\title{
14
}

\section{Moment Approximations of Individual-based Models}

\author{
Richard Law and Ulf Dieckmann
}

\subsection{Introduction}

This chapter illustrates insights into individual-based spatial models of ecological communities that can be gained from deterministic approximations. To do this we revisit some of the issues raised in Chapter 1 and show how approximations can help to

- separate the signal of an ecological stochastic process from intrinsic random variation;

- clarify qualitative dependencies that underlie the ecological stochastic process;

- determine how ecological stochastic processes depend on their parameters.

The deterministic approximations we use are the dynamics of spatial moments. These are closely related to pair-approximation methods (Matsuda et al. 1992; Harada and Iwasa 1994; see also Chapters 13, 18, and 19), but are constructed in a continuous rather than a discrete space. They also differ from diffusion approximations (see Chapters 16, 17, 22, and 23) in that they deal specifically with both structure at small spatial scales and the discrete nature of individual plants and animals. Moment methods represent a new departure in ecology for understanding the effects of interactions and movements of individuals in small neighborhoods (Bolker and Pacala 1997; Dieckmann et al. 1997; Law and Dieckmann, in press), and we argue that they hold promise for gaining understanding of ecological processes where the mean-field assumption breaks down. Moment methods are particularly helpful for providing insight into dynamics of plant communities, because interactions mostly occur with immediate neighbors (see Chapter 2). The methods also have potential for describing ecological dynamics in certain kinds of structured landscapes (Wiens et al. 1993; Dunning et al. 1995). 
Formal treatments of moment methods are given in Chapters 20 and 21. The equations used here are derived in Chapter 21 and differ in certain respects from those in Chapter 20. The motivation, however, is the same: to gain insight into the complex behavior of individual-based stochastic processes of ecological communities. We develop ideas in the context of plant communities comprising one or two competing species (see also Chapter 20), but the formal structures can be applied more widely to ecological systems with spatial structure.

\subsection{Spatial Patterns and Spatial Moments}

Consider a community living in a large, two-dimensional space, with individuals located at points $x=\left(x_{1}, x_{2}\right)$ in this plane. The abiotic environment is homogeneous in space, and any spatial structure that develops is generated internally by the community. Locations of individuals of species $i$ at some point in time $t$ are given by a function $p_{i}(x)$, and these are collected into a vector of density functions $p(x)=\left(p_{1}(x), p_{2}(x), \ldots\right)$ to give what we call the spatial pattern of the community. If individuals are not located at random in the plane (i.e., if their pattern is not described by a homogeneous Poisson process: see Chapter 5), we refer to the community as having spatial structure. As explained in Boxes 21.2 and 21.3, an individual is represented as a Dirac delta function, and the function $p_{i}(x)$ is the sum of all these individual contributions.

The community changes through three primary, stochastic events acting on individuals: birth, death, and movement. Whenever an event occurs, a new spatial pattern $p(x)$ is generated. Depending on how the events take place, a myriad of different spatial patterns can develop over the course of time; Figure 14.1 shows results at two points in time from just one realization of two competing species. The upper spatial pattern corresponds to a random layout of individuals of two species at time 0 . During the realization, the spatial pattern is repeatedly updated, and the lower spatial pattern in Figure 14.1 shows the pattern that has emerged by time 15, after approximately 2000 events have taken place. By this time, species 1 has developed aggregations of individuals and there is some spatial segregation such that where species 1 occurs, species 2 tends to be absent.

The problem with such realizations is that they are time consuming to generate and difficult to understand. Approximations based on moments try to avoid such drawbacks by replacing the spatial pattern with statistics summarizing its main features and then describing dynamics in terms of these statistics. In a sense this approach has been used in ecology for 

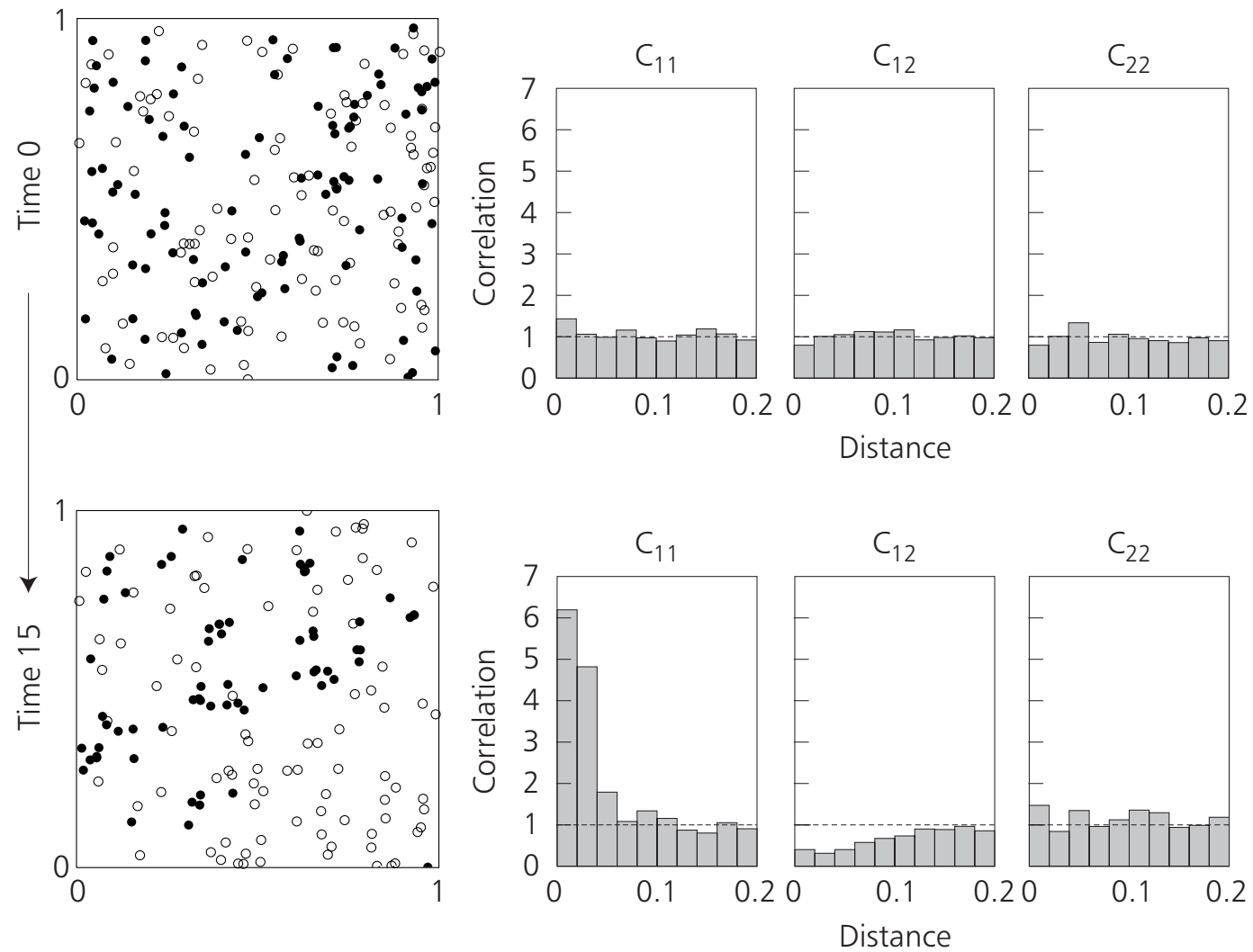

Figure 14.1 Spatial patterns at time 0 (top row) and time 15 (bottom row) from a realization of a stochastic model of two competing species. Locations of species 1 are given by filled circles; those of species 2 , by open circles. On the right-hand side are the three radial pair correlation densities that summarize important aspects of spatial information.

many years, because the spatial mean density, widely used by ecologists, is the lowest-order spatial moment. The new departure is putting into place dynamics of a second-order spatial moment that carries information about spatial structure over time.

The two spatial moments we use are defined as follows. The first moment,

$$
N_{i}(p)=\frac{1}{A} \int p_{i}(x) d x,
$$

is the familiar mean density of species $i$ over a spatial region $A$. The second moment, holding information on spatial structure, we call the pair correlation density function. This is a product of pairs of densities of individuals of species $i$ and $j$, averaged over the spatial region $A$ :

$$
C_{i j}(\xi, p)=\frac{1}{A} \int p_{i}(x)\left[p_{j}(x+\xi)-\delta_{i j} \delta(\xi)\right] d x,
$$

where $j$ is located at $\xi$ relative to $i$. [The term $\delta_{i j} \delta(\xi)$ is needed to remove self-pairs, and comprises the Kronecker symbol $\delta_{i j}$, which takes the 
value 1 when $i=j$ and 0 otherwise, and the Dirac delta function $\delta(\xi)$; see Boxes 21.2 and 21.3 and Section 21.3, Corrected correlation densities.] We scale the pair correlation densities here and below by dividing them by the product $N_{i} N_{j}$; in the absence of spatial structure they then take the value 1 ; aggregated (respectively regular) patterns are given by values greater (respectively less) than 1 . To make the functions still easier to depict, we display them in a radial form; this entails integrating over the angle around the circle, which results in no loss of information if the processes, like many of those in real ecological systems, are isotropic. The pair correlation density is not a central moment (see Chapter 20); we use it because the dynamic equations are then simpler (Chapter 21). It is important to understand that the second moment is a function rather than a scalar quantity, giving information on spatial structure over a range of spatial scales, in keeping with measures of spatial structure used in the past in plant ecology (Pielou 1968; Greig-Smith 1983).

To illustrate how pair correlation densities capture information about spatial structure, we have computed the second moments for the spatial patterns shown in Figure 14.1. In this two-species system there are three correlation functions associated with any spatial pattern: two auto-correlation functions $C_{11}(\xi, p), C_{22}(\xi, p)$, and a cross-correlation function $C_{12}(\xi, p)$ (Figure 14.1). At time 0 , there is no spatial structure and the functions are all flat. At time 15, the tendency for species 1 to form aggregations causes an excess of pairs at small distances, giving values of $C_{11}(\xi, p)$ much greater than 1 at short distances. Conversely, the segregation between species 1 and 2 causes a shortage of pairs at small distances, giving values of $C_{12}(\xi, p)$ much less than 1 at small distances.

During a realization of a stochastic process, the spatial moments (14.1) and (14.2) take new values each time the pattern changes. But, a realization is, of course, just one of an infinite ensemble that can be generated from the stochastic process, and it is the generic properties of the ensemble, not the properties of an individual realization, that we need to base our understanding on. We therefore replace the moments of the pattern at time $t$ with their averages over all realizations at this time:

$$
\begin{aligned}
& N_{i}=\int N_{i}(p) P(p) d p, \\
& C_{i j}(\xi)=\int P(p) C_{i j}(\xi, p) d p,
\end{aligned}
$$

where $P(p)$ is the probability density for patterns $p$ at time $t$, and the integration $d p$ is over the space of functions $p$. 
The quantities in Equations (14.3) and (14.4) comprise the state variables of the moment dynamics we use below. We derive a system of equations for these dynamics in Chapter 21, and these equations couple the changes in the first and second moments. In this way there is feedback from the spatial structure to the dynamics of the mean density (and vice versa) as the spatial system unfolds over time.

\subsection{Extracting the Ecological Signal from Stochastic Realizations}

Stochastic processes are a good framework in which to formalize ideas about ecological events acting on individuals (e.g., Pacala et al. 1996). In particular, they have the advantage of not glossing over the effects fluctuations have on local and global states of ecological systems. They have the drawback that it may not be obvious from individual realizations what the generic behavior of the process is. To illustrate this problem, and to show how moment dynamics can help to overcome it, we give an example of two competing plant species from Law and Dieckmann (in press), essentially a spatial version of the familiar Lotka-Volterra model of competition:

$$
\frac{d}{d t} N_{i}=\left(b_{i}-d_{i}\right) N_{i}-\sum_{j} d_{i j}^{\prime} N_{i} N_{j} \quad \text { for } i, j=1,2 .
$$

The equations are parameterized here with $b_{i}$ (respectively $d_{i}$ ) as a densityindependent birth (respectively death) rate. The term $d_{i j}^{\prime}$ is a component of the death rate that depends on the density of the competing species $j$, competition being intraspecific when $i=j$, and interspecific when $i \neq j$.

The spatial extension arises in that individuals are indexed by location in space, and the spatial model keeps track of (1) competition among individuals located close enough together and (2) movements of individuals. As we are dealing with plant species, we assume that movements are always associated with seed dispersal (birth of a new plant). Parameters of the community are set such that, on the one hand, species 1 is the stronger competitor and would replace species 2 in the absence of spatial structure. On the other hand, seeds of species 2 disperse over longer distances. Once the dynamics allow for spatial structure, whether species 2 goes to extinction is an open question.

Stochastic process. From the general birth-death-movement process given in Chapter 21 [Equations (21.1) and (21.2)], we can write down a stochastic process explicitly for two competing species. The probability per unit time of the transition from a pattern $p(x)$ to another pattern $p^{\prime}(x)$ is fully defined in terms of two independent events. 
- Birth. The probability per unit time $B_{i}\left(x, x^{\prime}, p\right)$ that an individual of species $i$, located at point $x$ in a pattern $p(x)$, gives rise to a daughter at location $x^{\prime}$ is given by

$$
B_{i}\left(x, x^{\prime}, p\right)=b_{i} m_{i}^{(b)}\left(x^{\prime}-x\right),
$$

where $b_{i}$, the birth rate, is multiplied by a dispersal term $m_{i}^{(b)}\left(x^{\prime}-x\right)$ independent of the birth rate, placing the daughter at location $x^{\prime}$ with probability density $m_{i}^{(b)}\left(x^{\prime}-x\right)$.

- Death. The probability per unit time $D_{i}(x, p)$ that an individual of species $i$, located at point $x$ in a pattern $p(x)$ dies is given by

$$
D_{i}(x, p)=d_{i}+\sum_{j} d_{i j}^{\prime} \int w_{i j}^{(d)}\left(x^{\prime}-x\right)\left[p_{j}\left(x^{\prime}\right)-\delta_{i j} \delta_{x}\left(x^{\prime}\right)\right] d x^{\prime} .
$$

Here, $d_{i}$ is the neighbor-independent component of the death rate common to all individuals, and the remaining terms on the right-hand side deal with the effects of competition with neighbors. Specifically, $w_{i j}^{(d)}\left(x^{\prime}-x\right)$ is a competition kernel (see Box 20.1) weighting the effect of a neighbor of species $j$ at location $x^{\prime}$ according to its distance from $x$; this is multiplied by the density $p_{j}\left(x^{\prime}\right)$ of plants of species $j$ at $x^{\prime}$, integrated over all locations $x^{\prime}$, and scaled by the parameter $d_{i j}^{\prime}$. The expression $\delta_{i j} \delta_{x}\left(x^{\prime}\right)$ removes the target plant of species $i$ at location $x$ from the competition kernel; it is needed because the target plant does not compete with itself.

We use Gaussian functions to describe dispersal distances and the effect of distance on competition, and characterize the functions with two parameters. The first parameter $s_{i j}^{(b)}$ (respectively $s_{i j}^{(d)}$ ) is the standard deviation for dispersal (respectively competition). The second parameter truncates the functions at maximum radius $r_{i j}^{(b)}$ (respectively $r_{i j}^{(d)}$ ) for dispersal (respectively competition), and can be thought of as setting an upper limit on the distance over which dispersal (respectively competition) takes place. The functions are normalized so that their integrals are equal to 1. Table 14.1 gives the parameter values; notice the competition parameters are set such that species 1 is a stronger competitor than species 2, but disperses over shorter distances than species 2 .

Four realizations of the stochastic process, run from time 0 to 15 , are shown in Figure 14.2. (Here, and in all realizations below, the stochastic process is implemented in a space of unit area with periodic boundaries.) The graphs give the paths in the plane of population densities of the two species, and it is important not to confuse this with the physical space in 
Table 14.1 Parameter values for a community of two competing species. Source: Law and Dieckmann (in press).

$\begin{array}{lllll}\hline & & \multicolumn{2}{l}{\text { Value for species } i} & \\$\cline { 3 - 4 } & \text {$\left.Parameter } & i=1 & i=2 & \text { Explanation } \\ \hline \text { Death } & d_{i} & 0.2 & 0.2 & \\ & d_{i 1}^{\prime} & 0.001 & 0.002 \\ & d_{i 2}^{\prime} & 0.0005 & 0.001\end{array}\right\} \quad \begin{aligned} & \text { Species 1 is a stronger } \\ & \text { competitor than species 2 } \\ & \end{aligned}$

Note: Simulations were done on the unit square with periodic boundaries.

which the two species live and interact. The generic behavior, or signal, of the stochastic process is not clear from inspection of the paths. Demographic stochasticity masks any obvious trend: the paths differ from one another and have the appearance of tangled webs. As one would expect, matters are improved by taking the mean path of some realizations, but the number of realizations needed may be quite large. This can be seen in Figure $14.2 \mathrm{e}$, which shows the mean of 20 realizations; a distinct curl to the path is now evident.

Moment dynamics. From the general equations for the dynamics of the first and second spatial moments (21.9) and (21.10), we can write down the equations as they apply to two competing species. The dynamics of the first moments (mean densities) are given by

$$
\frac{d}{d t} N_{i}=\left(b_{i}-d_{i}\right) N_{i}-\sum_{j} d_{i j}^{\prime} \int w_{i j}^{(d)}\left(\xi^{\prime}\right) C_{i j}\left(\xi^{\prime}\right) d \xi^{\prime},
$$

for $i, j=1,2$. The first term on the right-hand side is the neighborhoodindependent component of births and deaths, and the second term is the neighborhood-dependent component of deaths. Evidently, in turning from the nonspatial version of the Lotka-Volterra equations to the spatial version, the products $N_{i} N_{j}$ of the Lotka-Volterra equations are replaced by integrals involving the pair correlation densities, compare Equations (14.5) and (14.8). The effect of competition depends fundamentally on the relative 
(a)

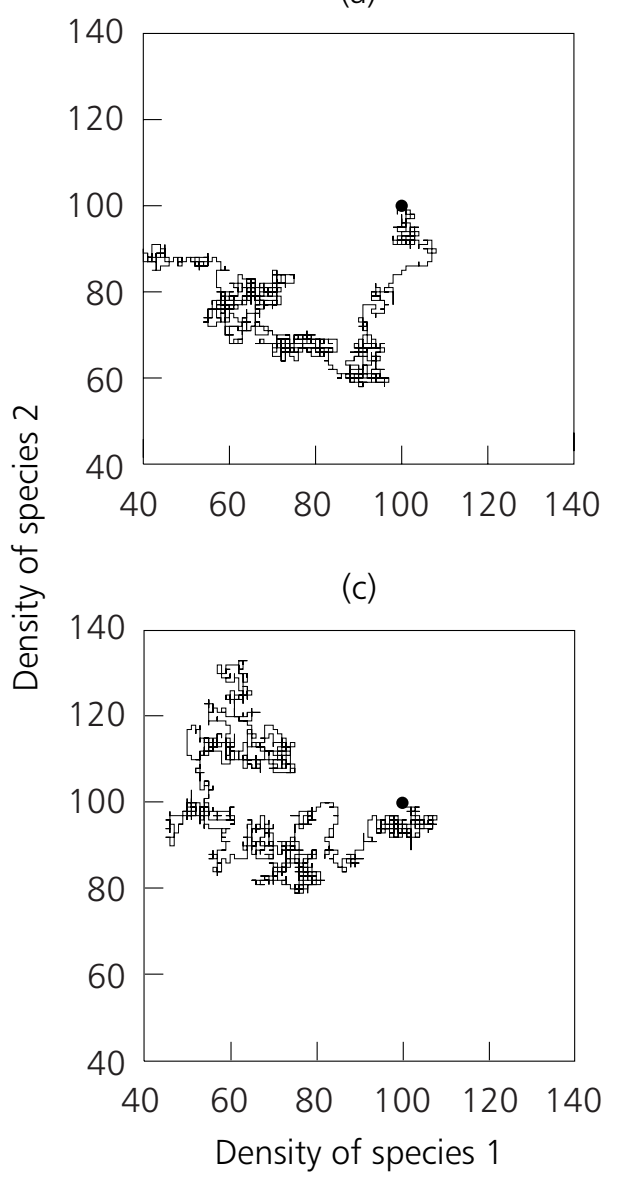

(b)

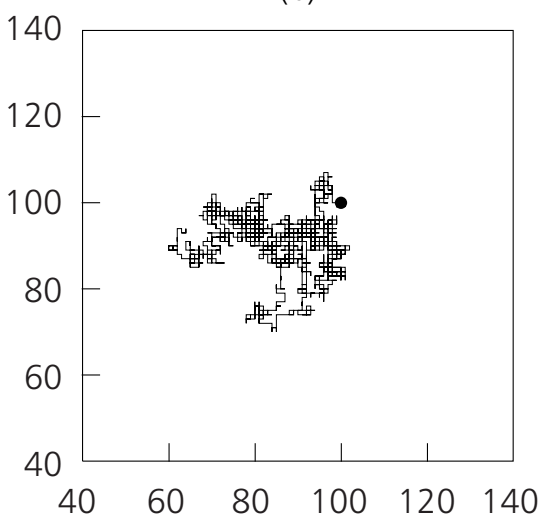

(d)

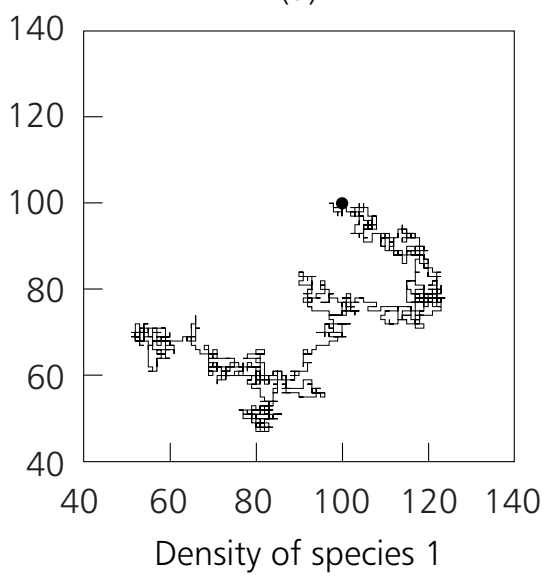

(e)

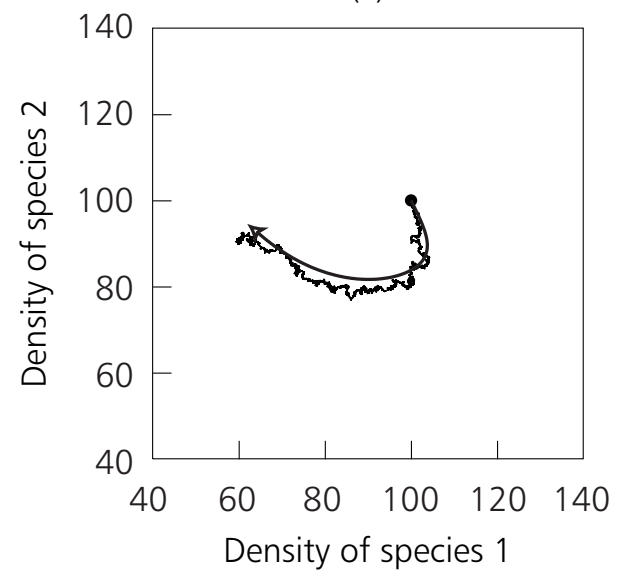

Figure 14.2 Plane of population density of two competing species. Graphs (a), (b), (c), and (d) show the path mapped out by individual stochastic realizations over 15 time units. (e) Mean path of 20 realizations, with the deterministic approximation (found by solving the equations for the dynamics of the first and second spatial moments) superimposed as a smooth curve. The filled circle marks the starting point of each path. 
magnitude of these integrals, and we should therefore expect the outcome to be radically altered by the introduction of spatial structure.

The dynamics of the second moment (pair correlation densities) are given by

$$
\begin{aligned}
& \frac{d}{d t} C_{i j}(\xi)= \\
& +b_{i} \int m_{i}^{(b)}\left(\xi^{\prime}\right) C_{i j}\left(\xi+\xi^{\prime}\right) d \xi^{\prime}+b_{j} \int m_{j}^{(b)}\left(\xi^{\prime}\right) C_{j i}\left(-\xi+\xi^{\prime}\right) d \xi^{\prime} \\
& +\delta_{i j} b_{i} m_{i}^{(b)}(-\xi) N_{i} \quad+\delta_{j i} b_{j} m_{j}^{(b)}(\xi) N_{j} \\
& -d_{i} C_{i j}(\xi) \quad-d_{j} C_{j i}(-\xi) \\
& -\sum_{k} d_{i k}^{\prime} \int w_{i k}^{(d)}\left(\xi^{\prime}\right) T_{i j k}\left(\xi, \xi^{\prime}\right) d \xi^{\prime}-\sum_{k} d_{j k}^{\prime} \int w_{j k}^{(d)}\left(\xi^{\prime}\right) T_{j i k}\left(-\xi, \xi^{\prime}\right) d \xi^{\prime} \\
& -d_{i j}^{\prime} w_{i j}^{(d)}(\xi) C_{i j}(\xi) \quad-d_{j i}^{\prime} w_{j i}^{(d)}(-\xi) C_{j i}(-\xi),
\end{aligned}
$$

for $i, j=1,2$. The term $\delta_{i j}$ is the Kronecker symbol, and $T_{i j k}\left(\xi, \xi^{\prime}\right)$ is the correlation density of triplets, for which we assume the moment closure

$$
T_{i j k}\left(\xi, \xi^{\prime}\right)=\frac{1}{N_{i}} C_{i j}(\xi) C_{i k}\left(\xi^{\prime}\right),
$$

as explained in Section 21.4. With 10 terms, some of which are integrals, Equation (14.9) looks somewhat forbidding. But the complexity of the equation is not altogether surprising, because there are five types of event in species $i$ affecting the flux in and out of the pair density $C_{i j}(\xi)$, as given by the terms in the first column on the right-hand side of Equation (14.9). For each type of event affecting $i$, there is an equivalent one affecting $j$, given by the terms in the second column. It can be seen from inspection of Equations (14.8) and (14.9) that they are coupled, so changes in the mean density affect the rate of change of the pair correlation densities, and vice versa.

Numerical integration of Equations (14.8) and (14.9) clearly indicates the signal of the stochastic process, shown as the smooth curve in Figure 14.2e. At the start, species 1 is favored over species 2, but, after a short period of time, this is reversed. The reason for this reversal is quite interesting and results from the generation of spatial structure in the community (Law and Dieckmann, in press). To begin with, individuals are situated at random locations in the plane. Species 1, the stronger competitor, is at an advantage in these circumstances and starts to increase in density, whereas species 2 decreases; thus the deterministic path starts by pointing down and to the right. But species 1 is also a poorer disperser and begins to develop a clumped spatial pattern which inflates the strength of intra-specific competition. Eventually, clumping becomes strong enough to place species 1 at 
a disadvantage in the community, causing the deterministic path to swing round in the direction of increasing density of species 2 and decreasing density of species 1 .

The deterministic path in Figure 14.2e provides information on a small part of the plane of population densities. To illustrate the dynamical behavior more broadly, we have taken a larger sample of paths and run them for a longer period of time, as shown in Figure 14.3 (Law and Dieckmann, in press). Figure 14.3a gives the mean path, averaged over 20 realizations, from a grid of starting points of the stochastic process. This shows that species 1 , despite its competitive advantage, is eventually always driven to extinction due to the excess intraspecific competition it generates by spatial aggregation. Figure $14.3 \mathrm{~b}$ gives the corresponding deterministic paths obtained by solving Equations (14.8) and (14.9). There is a close match between the paths in Figure 14.3a and 14.3b: evidently the moment dynamics give a good approximation to the behavior of the stochastic process. The same cannot be said of the nonspatial Lotka-Volterra competition equations (14.5), shown in Figure 14.3c. These equations neglect the effects of dispersal and the small neighborhoods within which competition occurs, and lead one to expect that species 2 should be driven to extinction. Space is clearly crucial here - to ignore it is to be qualitatively in error.

The message from this analysis of two competing species is threefold. (1) The signal of the stochastic process often can barely be seen from looking at individual realizations. (2) The method of moments provides a close approximation to the average behavior of the stochastic process and can be used to gain insight into its generic features. (3) The spatial extension is fundamental to understanding the dynamics of plants competing and dispersing in small neighborhoods.

\subsection{Qualitative Dependencies in a Spatial Logistic Equation}

Here we show some qualitative properties of stochastic realizations that can be better understood by means of deterministic approximations. We do this in the context of a spatial extension of the familiar logistic model of single-species population growth

$$
\frac{d}{d t} N=(b-d) N-d^{\prime} N^{2}
$$

parameterized in the same way as Equations (14.5). The spatial extension turns out to have some qualitative features that are surprising, at least at first 
(a)

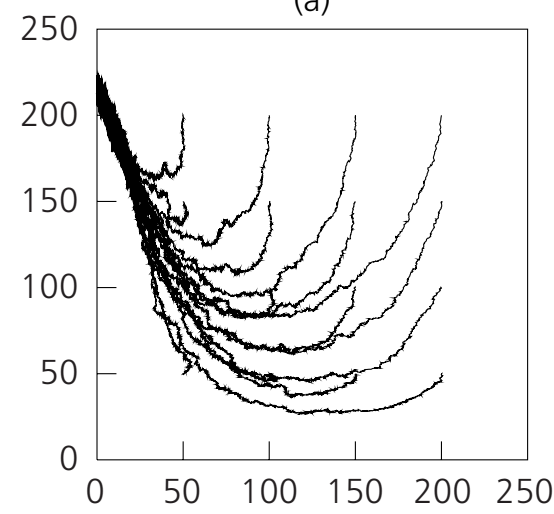

(b)

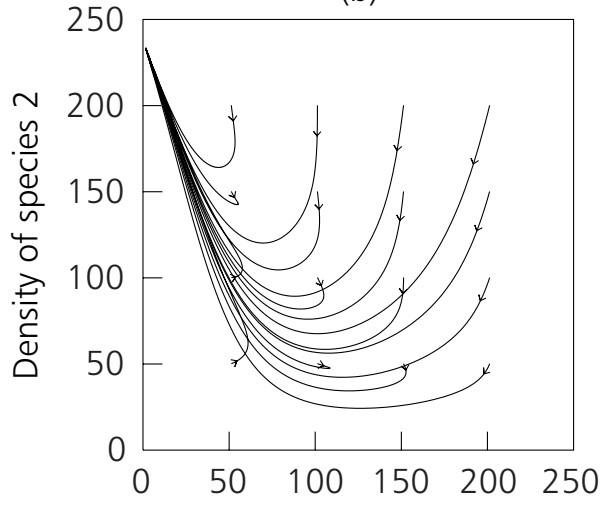

(c)

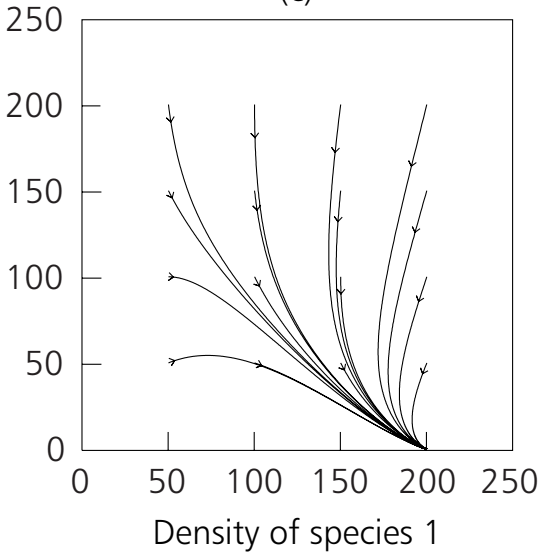

Figure 14.3 Plane of population density of two competing species. Paths are given from a grid of 16 combinations of starting densities. Individuals are placed at random locations in space at the start, and paths are allowed to run for 100 time units. (a) Mean paths of 20 realizations of the stochastic process. (b) Deterministic paths given by the dynamics of the first and second moments. The paths are projections from higher-dimensional dynamics, Equations (14.8) and (14.9), and may therefore intersect one another. (c) Paths given by the nonspatial, Lotka-Volterra competition equations. Source: Law and Dieckmann (in press). 
sight. Yet these features follow in a simple, natural way from the moment dynamics, as we show below.

Stochastic process. The stochastic process has much in common with the spatial Lotka-Volterra competition equation described in Section 14.3, allowing (1) an increased risk of death of individuals located close enough together and (2) dispersal of seeds. Notationally, the stochastic process is easier to handle because, with only one species, terms do not need to be indexed by species. As before, there are two stochastic events.

- Birth. The probability per unit time $B\left(x, x^{\prime}, p\right)$ that a plant, located at point $x$ in a pattern $p(x)$, gives rise to a daughter plant at location $x^{\prime}$ is given by

$$
B\left(x, x^{\prime}, p\right)=b m^{(b)}\left(x^{\prime}-x\right)
$$

Death. The probability per unit time $D(x, p)$ that a plant, located at $x$ in a pattern $p(x)$, dies is given by

$$
D(x, p)=d+d^{\prime} \int w^{(d)}\left(x^{\prime}-x\right)\left[p\left(x^{\prime}\right)-\delta_{x}\left(x^{\prime}\right)\right] d x^{\prime} .
$$

Terms are as defined in Equations (14.6) and (14.7), and parameter values that remain fixed in the simulations below are $b=0.4, d=0.2$, and $d^{\prime}=0.001$.

Three sets of realizations of this stochastic process are given in Figure 14.4. The sets differ in the size of the competition neighborhoods and in the distances over which seeds disperse. The results are surprising. Depending on the choice of parameter values for local competition and seed dispersal, the populations may grow to densities substantially larger or smaller than one would expect from the equilibrium density of the logistic equation (14.11), which equals 200 with the parameter values above. This is a good example of the new phenomena that emerge when the spatial extension to population dynamics is introduced, as has been stressed at many places in Part B.

Moment dynamics. Why does the asymptotic density depend on the competition neighborhood and distances over which seeds disperse? The answer becomes apparent once the equation for the dynamics of the first spatial moment of the stochastic process is put into place; this is a simplified version of Equations (14.8),

$$
\frac{d}{d t} N=(b-d) N-d^{\prime} \int w^{(d)}\left(\xi^{\prime}\right) C\left(\xi^{\prime}\right) d \xi^{\prime} .
$$


(a)

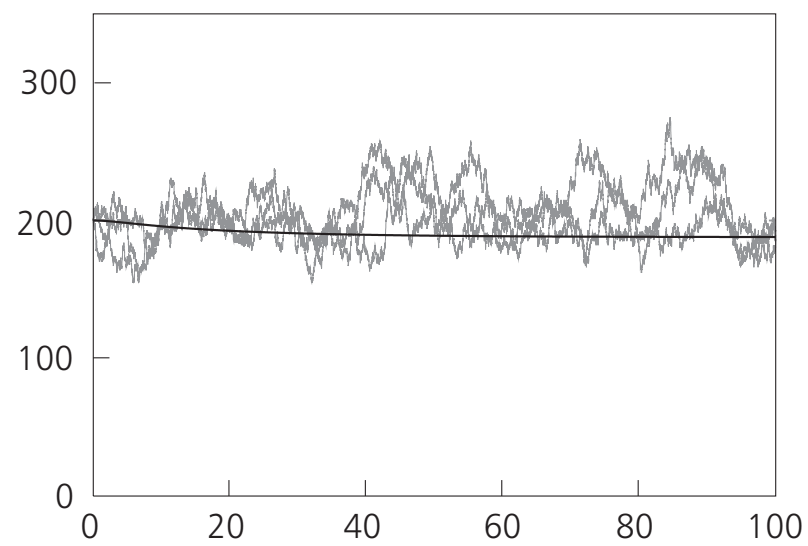

(b)

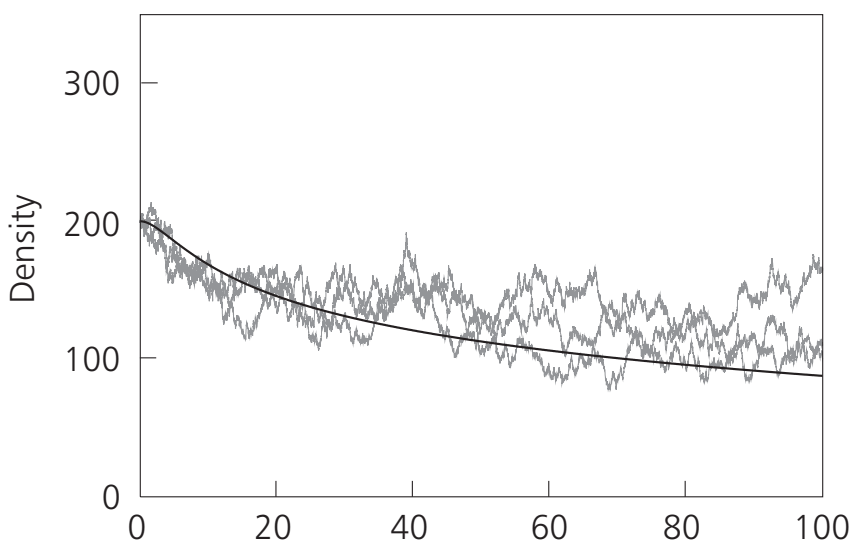

(c)

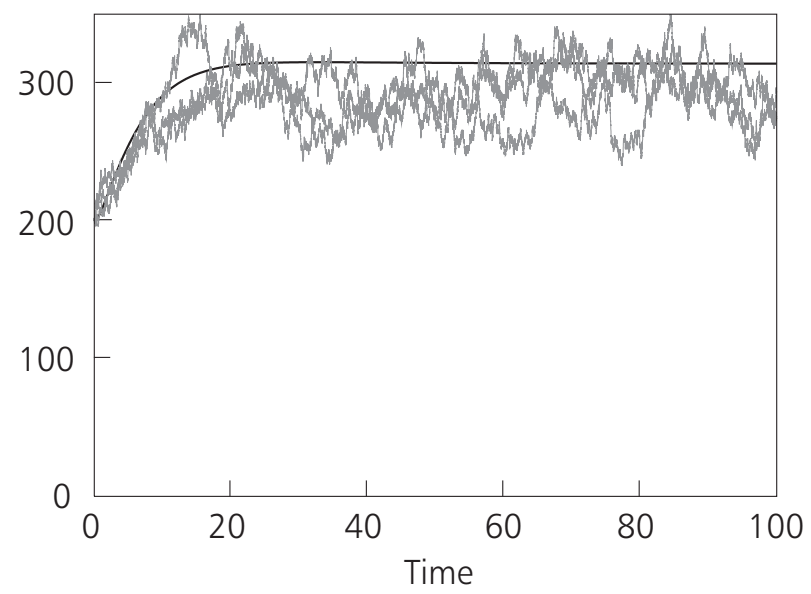

Figure 14.4 Stochastic realizations of a spatial, logistic model of population growth with contrasting dispersal and competition neighborhoods. In each case three realizations are run, starting with 200 individuals located at random (200 is the equilibrium value of the corresponding nonspatial logistic model). (a) Competition and dispersal take place over large distances: $s^{(d)}=0.15, s^{(b)}=0.15$. (b) Competition and dispersal take place over intermediate distances: $s^{(d)}=0.05, s^{(b)}=0.05$. (c) Competition takes place at a smaller scale than dispersal: $s^{(d)}=0.015, s^{(b)}=0.15$. The maximum radii are three times the standard deviations. Superimposed on the realizations is the deterministic approximation obtained from solving the equations for the dynamics of the first and second moments. 
The term $N^{2}$ of the nonspatial model is replaced by an integral involving the pair correlation densities, compare Equations (14.11) and (14.14). Evidently, the outcome depends on the spatial structure that develops over time, as given by $C\left(\xi^{\prime}\right)$, and on the extent to which plants experience this structure, as given by $w^{(d)}\left(\xi^{\prime}\right)$. If the integral is greater than $N^{2}$, the population comes to equilibrium at a density less than that of the nonspatial version. Conversely, if the integral is less than $N^{2}$, the equilibrium density is greater than that of the nonspatial version. Clearly, details of the spatial structure really do matter.

To understand how the spatial structure develops, we need the dynamics of the second moment:

$$
\begin{aligned}
\frac{1}{2} \frac{d}{d t} C(\xi)= & +b \int m^{(b)}\left(\xi^{\prime \prime}\right) C\left(\xi+\xi^{\prime \prime}\right) d \xi^{\prime \prime} \\
& +b m^{(b)}(\xi) N \\
& -d C(\xi) \\
& -d^{\prime} C(\xi) \int w^{(d)}\left(\xi^{\prime}\right) C\left(\xi^{\prime}\right) / N d \xi^{\prime} \\
& -d^{\prime} w^{(d)}(\xi) C(\xi) .
\end{aligned}
$$

This equation is simplified from Equation (14.9). The two columns on the right-hand side of Equation (14.9) can be added together, because there is only one species and the process is isotropic; we then divide both sides by 2 , getting the factor $1 / 2$ on the left-hand side of Equation (14.15).

It turns out that the spatial structure that develops over time depends on the size of the competition neighborhood and dispersal distances. Figure 14.5 illustrates this with stochastic realizations using the three combinations of parameter values given in Figure 14.4. All three start with the same random spatial pattern and show the spatial pattern of a realization of the stochastic process after a long period of time has elapsed. In Figure 14.5a, seeds disperse over relatively large distances and the correlation function shows rather little spatial structure. In fact, it would not make much difference if there were more small-scale spatial structure, because the neighborhoods of competition integrate over relatively large areas and would be blind to such structure. In Figure 14.5b, where both dispersal distances and competition neighborhoods are smaller, matters are different. Shorter dispersal leads to more aggregation, and the smaller neighborhoods of competition make the plants sensitive to this structure. Thus the plants experience a density much greater than the spatial average in the interaction neighborhoods, and population growth is stopped at lower mean density. In 


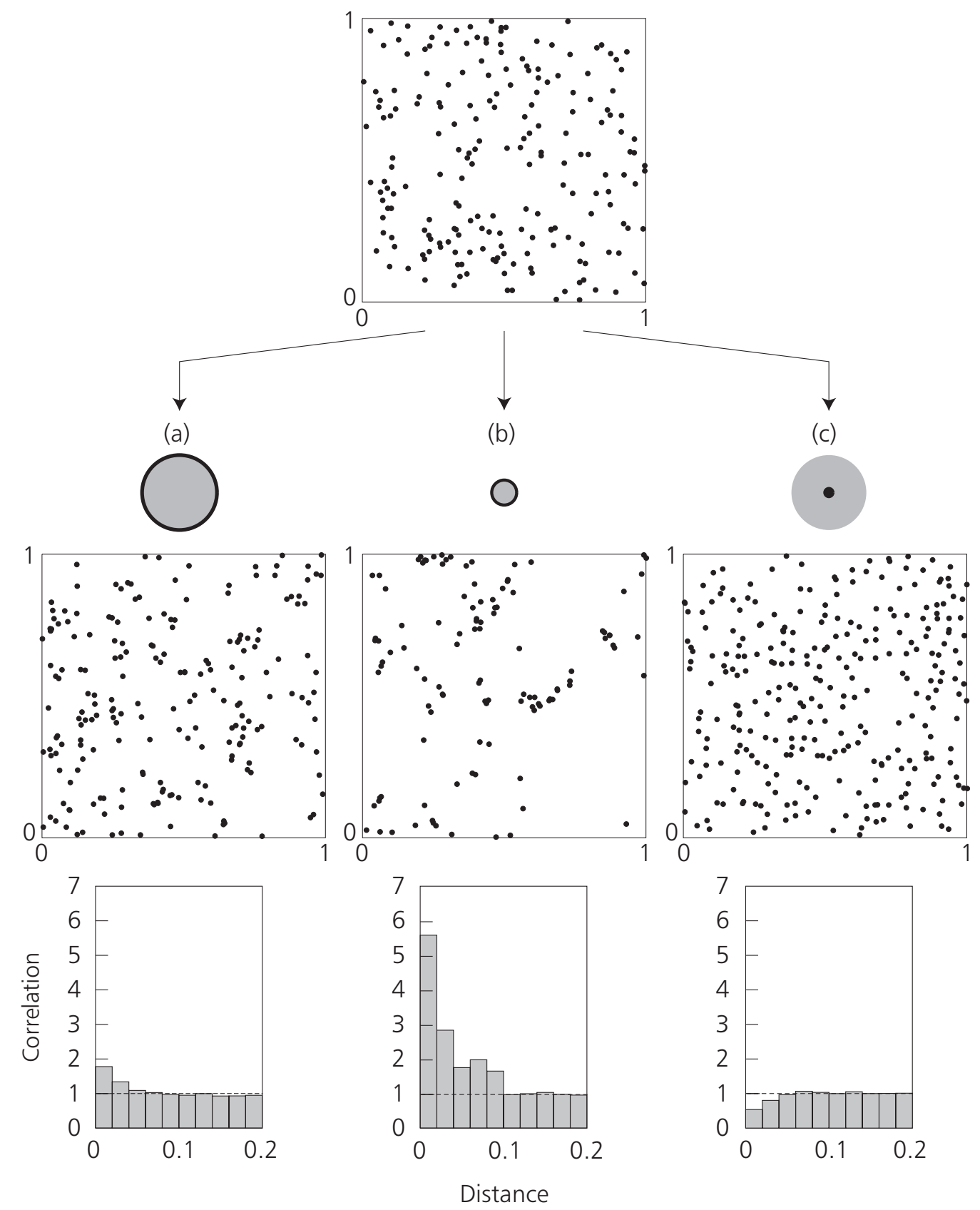

Figure 14.5 Effect of contrasting dispersal and competition neighborhoods on spatial pattern in a logistic model of population growth. The spatial pattern at the top shows the initial state, comprising 200 individuals placed at random locations. Columns (a), (b), and (c) have parameter values matching Figures $14.4 \mathrm{a}, 14.4 \mathrm{~b}$, and $14.4 \mathrm{c}$, respectively; the radius of the heavy circle is the standard deviation $s^{(b)}$ of the competition neighborhood, and the radius of the shaded area is the standard deviation $s^{(d)}$ of the dispersal neighborhood. The columns show the state of sample realizations at time 100 in terms of the spatial pattern and the corresponding auto-correlation function. 
Figure $14.5 \mathrm{c}$, the competition neighborhoods are made still smaller (while the dispersal neighborhood is reset to a larger size), and plants that lie too close together have very high mortality rates. This high mortality of plants with close neighbors leads to a regular spatial pattern at short distances. The correlation function is now much less than 1 in the immediate neighborhood of plants, and the plants experience a local density much lower than the spatial average. The population therefore continues to grow to a greater mean density.

How good an approximation to the stochastic process do the moment dynamics provide? A partial answer is given by placing the time course of mean density on the stochastic realizations in Figure 14.4. This shows that the moment dynamics do correctly capture the qualitative outcome and give a reasonable approximation to the quantitative properties of the dynamics.

The message from this example is that qualitative features of the stochastic realizations, at first sight surprising and non-intuitive, become much easier to understand once dynamics have been reduced to the first and second spatial moments. As an ecological footnote, the logistic equation, especially its equilibrium density, has played a central role in developing ideas in ecology. The results here suggest that we may be seriously misled in applying the nonspatial logistic equation to systems with strong spatial structure and small neighborhoods of interaction (see Roughgarden 1997). We should stress though that moment models have still to address some important properties of population growth, such as the dependence of competition neighborhood on size of individuals (Chapter 2).

\subsection{Exploration of Parameter Space}

The results in Section 14.4 suggest that the spatial extension of the logistic equation has dynamics that depend sensitively on the size of the neighborhoods over which individuals compete and the distances over which dispersal occurs. But so far we have only investigated three fixed points in parameter space and have little idea about the overall form of the dependence. Below, we use the deterministic approximation based on the dynamics of the first and second spatial moments, Equations (14.14) and (14.15), to show how more knowledge about the equilibrium density can be gained.

A convenient method for obtaining equilibrium densities of the moment dynamics is 
(a)

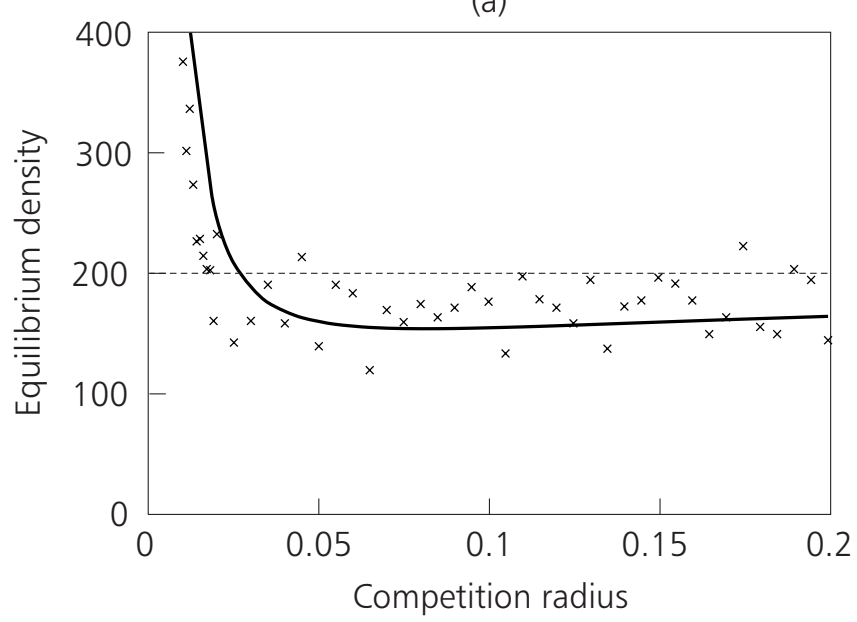

(b)

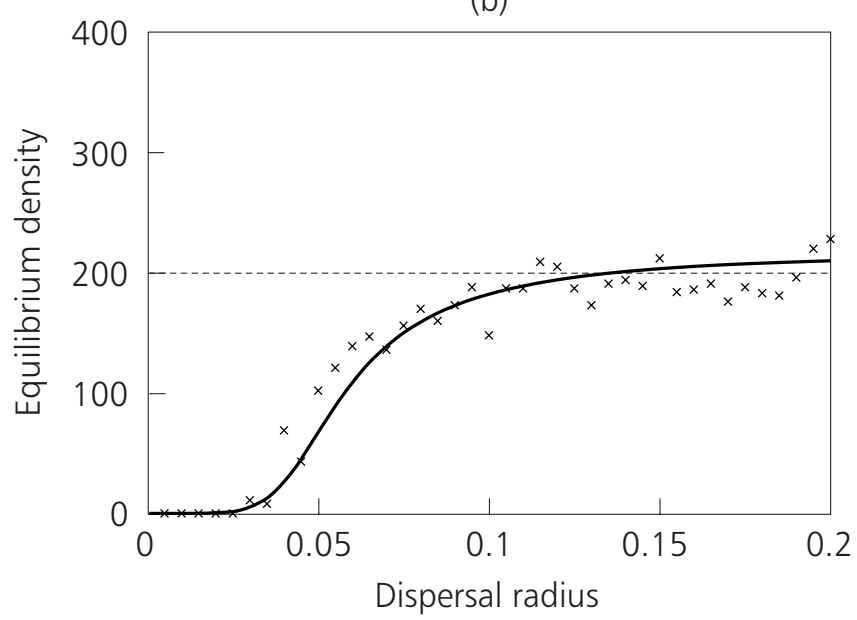

Figure 14.6 Equilibrium densities of a spatial logistic equation, expressed as a function of competition radius and dispersal distance. (a) Competition radius $s^{(d)}$ is varied between 0 and 0.2 for $s^{(b)}=0.08$; (b) shows the effect of varying $s^{(b)}$ over the same interval for $s^{(d)}=0.05$. While outcomes of stochastic realizations (crosses) show considerable scatter, and mean-field results (dashed lines) are sometimes qualitatively in error, the deterministic approximation based on spatial moments (continuous curves) provides satisfactory descriptions of how equilibrium densities depend on the radii.

- to determine, with $\hat{c}(\xi)=\hat{C}(\xi) / \hat{N}^{2}$, the equilibrium of the first spatial moment for a given second moment from Equation (14.14),

$$
\hat{N}=\frac{b-d}{d^{\prime} \int w^{(d)}\left(\xi^{\prime}\right) \hat{c}\left(\xi^{\prime}\right) d \xi^{\prime}},
$$

- and then, using that solution in Equation (14.15), to solve numerically for the equilibrium of the (normalized) second moment, $\frac{d}{d t} \hat{c}(\xi)=0$.

Figure 14.6 shows how sensitive equilibrium densities are to changes in local competition and dispersal. Evidently, the equilibrium density given 
by the mean-field results of the nonspatial logistic equation applies only in the limits as the interaction neighborhood becomes large and dispersal distances become large. If the competition neighborhood is small relative to dispersal, densities much in excess of the mean-field value occur due to the tendency of other individuals to be absent in this small neighborhood (Figures 14.5c and 14.6a). At the other end of the scale, if dispersing offspring tend to fall within the competition neighborhood, and the competition neighborhood is itself small, the population can go extinct (left range in Figure 14.6b). Extinction comes about as a result of dense aggregations of individuals within the competition neighborhood. The denominator in Equation (14.16) is then large; for finite populations, this leads to population sizes so small that accidental extinction by demographic stochasticity becomes very likely.

Clearly, the spatial version of the logistic equation has some new and interesting features. But the new features that emerge are not easily accessible from numerical studies of the stochastic process itself. It is through approximation schemes, here the moment dynamics, that we gain understanding of how the dynamics depend on model parameters. Once a dynamical system that gives an acceptable approximation to the stochastic process is in place, a battery of analytical techniques (such as methods from bifurcation theory) is available to gain understanding of the consequences of spatial heterogeneity.

\subsection{Concluding Comments}

If a strong foundation for studying the dynamics of spatially structured communities is to be developed, we need methods that give good approximations to the underlying, intricate, individual-based processes. We think methods based on the dynamics of spatial moments hold promise in this regard. The approximations evidently work well. Moment models enable the signals from ecological stochastic processes to be extracted reliably and give new insights into the rich dynamics of spatial ecological processes. The models are, of course, no more than approximations and work best where spatial structure applies at a small spatial scale and is not too extreme in intensity; the range of spatial structures over which the method works has yet to be determined in detail. Structure at large spatial scales is better dealt with by diffusion approximations (Chapters 16, 17, 22, and 23); to account for spatial structure at both small and large scales simultaneously, it may be possible to amalgamate moment and diffusion approximations. 
A recurring feature of the moment dynamics above is the use of a spatial integral of the pair correlation densities weighted by some function of distance, Equations (14.8) and (14.9), in place of the product of the average densities $N_{i} N_{j}$. We are replacing the spatial average of the densities with what might be termed an average neighborhood that carries information about local spatial structure. The integral, in effect, formalizes a notion of the "plant's-eye view" of the community that has been in the plant-ecological literature for many years (Turkington and Harper 1979; Mahdi and Law 1987). In switching the focus to the average neighborhoods of plants, some basic changes in plant community dynamics are to be expected. For instance, it is well established in natural plant communities that conspecifics tend to be aggregated (e.g., Greig-Smith 1983; Mahdi and Law 1987). As shown in Chapter 20, spatial structure may make it much easier to achieve coexistence of plant species than has previously been thought.

Evidently, theoretical ecologists have some fundamental thinking to do about the effects of spatial structure. Many basic ideas in ecology come from theory based on the mean-field assumption: the maximum sustainable yield, the competitive exclusion principle, community stability, and so on. But terrestrial communities are spatially structured, and mean-field dynamics are often inappropriate. It is clear from the examples given here and elsewhere in the book that spatial structure needs to be properly incorporated into ecological dynamical systems if we are to avoid coming to seriously mistaken conclusions about ecological processes. Moment approximations provide a key for opening the door into spatial structure. 


\section{References}

References in the book in which this chapter is published are integrated in a single list, which appears on pp. 517-552. For the purpose of this reprint, references cited in the chapter have been assembled below.

Bolker B \& Pacala SW (1997). Using moment equations to understand stochastically driven spatial pattern formation in ecological systems. Theoretical Population Biology 52:179-197

Dieckmann U, Herben T \& Law R (1997). Spatio-temporal processes in plant communities. In Yearbook 1995/96, Institute for Advanced Study Berlin, ed. Lepenies W, pp. 296-326. Berlin, Germany: Nicolaische Verlagsbuchhandlung

Dunning JB Jr, Stewart DJ, Danielson BJ, Noon BR, Root TL, Lamberson RH \& Stevens EE (1995). Spatially explicit population models: Current forms and future uses. Ecological Applications 5:3-11

Greig-Smith P (1983). Quantitative Plant Ecology, 3rd edn. Oxford, UK: Blackwell

Harada Y \& Iwasa Y (1994). Lattice population dynamics for plants with dispersing seeds and vegetative propagation. Researches on Population Ecology 36:237-249

Law R \& Dieckmann U. A dynamical system for neighborhoods in plant communities. Ecology. In press

Mahdi A \& Law R (1987). On the spatial organization of plant species in a limestone grassland community. Journal of Ecology 75:459-476

Matsuda HN, Ogita A, Sasaki A \& Satō K (1992). Statistical mechanics of population: The lattice Lotka-Volterra model. Progress in Theoretical Physics 88: $1035-1049$

Pacala SW, Canham CD, Saponara J, Silander JA Jr, Kobe RK \& Ribbens E (1996). Forest models defined by field measurements: Estimation, error analysis and dynamics. Ecological Monographs 66:1-43

Pielou EC (1968). An Introduction to Mathematical Ecology. New York, NY, USA: Wiley InterScience

Roughgarden J (1997). Production functions from ecological populations: A survey with emphasis on spatially implicit models. In Spatial Ecology: The Role of Space in Population Dynamics and Interspecific Interactions, eds. Tilman D \& Karieva P, pp. 296-317. Princeton, NJ, USA: Princeton University Press

Turkington RA \& Harper JL (1979). The growth, distribution and neighbour relationships of Trifolium repens in a permanent pasture. I. Ordination, pattern and contact. Journal of Ecology 67:201-218 